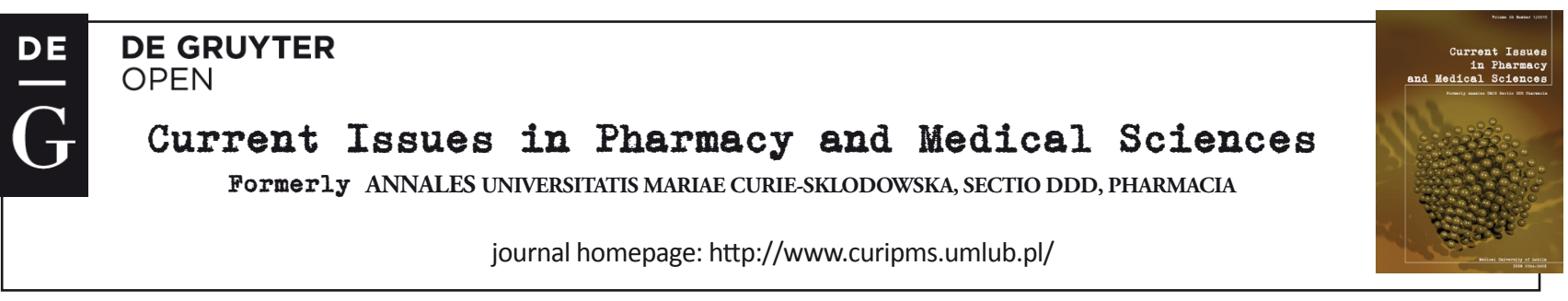

\title{
A brief analysis of patients suffering from stomach or duodenal ulcers in Almaty hospital № 1
}

\author{
Aliya Aryzbekova ${ }^{1}$, Konrad Tomasz Juszkiewicz ${ }^{2}$, Donald Eduard Burgess ${ }^{2}$, \\ Andrzej Polski ${ }^{3 *}$, Ewa PoleszaK ${ }^{3}$
}

\begin{abstract}
${ }^{1}$ Department of Microbiology, Virology and Immunology, Kazakh National Medical University, 88 Tole Bi, Almaty, Kazakhstan
${ }^{2}$ DRK Biomedical Research and Development LLC, 788 Los Alamos Avenue, Livermore, CA, USA

${ }^{3}$ Chair and Department of Applied Pharmacy, Faculty of Pharmacy, Medical University of Lublin, 1 Chodzki, 20-093 Lublin, Poland
\end{abstract}

\begin{tabular}{l} 
ARTICLE INFO \\
\hline Received 08 October 2015 \\
Accepted 06 November 2015
\end{tabular}

\section{Keywords:}

stomach ulcer,

duodenal ulcer,

hospital.

\begin{abstract}
Peptic ulcers are a serious problem worldwide, and affect about 4 million people each year. Their etiology is connected with the presence of Helicobacter pylori, the act of smoking, drinking alcohol, being stress, and taking excessively nonsteroidal anti-inflammatory drugs, as well as steroids. The most common symptoms are abdominal pain, nausea, chest pain and fatigue, while less frequent symptoms include vomiting and weight loss. Helicobacter pylori is responsible for about $80 \%$ of gastric and $90 \%$ of duodenal ulcer cases. In this work, an analysis is made of a correlation between stomach or duodenal ulcer and gender, residence and number of patients hospitalized in the Almaty hospital №1, from 2009-2012, in order to learn about trends in the incidence of these diseases in Kazakhstan. A total number of 950 patients with stomach and duodenal ulcers, in 2009-2012, were questioned. The patient's residence, gender and stomach or duodenal ulcer problem were taken into account in the study. The result of this work reveals that the largest amount of hospitalized patients suffering from stomach or duodenal ulcers came from urban areas. Moreover, more women than men suffered from peptic ulcers. Furthermore, the number of patients admitted to the hospital due to duodenal ulcers did not show any variation throughout the study. However, the least number of patients suffering from gastric ulcers was noticed in December 2009, and the greatest was in October and November 2011. The obtained data show that ulcers are a serious problem in Kazakhstan.
\end{abstract}

\section{INTRODUCTION}

Peptic ulcers can be classified as either being a stomach ulcer (gastric ulcer) or a duodenal ulcer. The last are found in the upper area of the small intestine. Peptic ulcers are a serious problem, affecting about 4 million people worldwide each year [18]. Women and older people are more susceptible, however, young men often suffer from duodenal ulcers, while stomach ulcers are more likely to occur in older women [16]. Their etiology is most often connected with the presence of Helicobacter pylori, the act of smoking, drinking alcohol excessively, feeling stressed, and taking in excess, nonsteroidal anti-inflammatory drugs, as well as steroids $[10,13]$. The most common symptoms of peptic ulcer are

\footnotetext{
^ Corresponding author

e-mail: andrzejpolski@umlub.pl

tel. +48814487040 , fax +48814487040
}

abdominal pain, nausea, chest pain and fatigue, while less frequent symptoms include vomiting and weight loss [12].

One of the most important factor responsible for ulcers is the presence of Helicobacter pylori. This was first identified by Barry J. Marshall and J. Robin Warren in 1982. In 2005, they were awarded the Nobel Prize in Physiology or Medicine for their study on "the bacterium Helicobacter pylori and its role in gastritis and peptic ulcer disease" [11]. H. pylori is a gram-negative helical shaped bacterium, that is present mainly in the stomach, but also in other human organs such as the eye, etc $[5,6]$.

The World Health Organization (WHO) estimates that approximately $70 \%$ of all people in developing countries, and approximately $30 \%$ of the entire population within developed countries are infected with $H$. pylori [8]. It is responsible for about $80 \%$ cases of all gastric ulcers and $90 \%$ cases of all duodenal ulcer cases. On the other hand, 
the majority of individuals infected by $H$. pylori do not develop this disease [2]. Of note: its presence increases the risk of peptic ulcers and gastritis type $\mathrm{B}$, which sometimes leads to cancer.

\section{AIM}

The main objective of the work was to derive a brief analysis of a potential correlation between being afflicted with a stomach or duodenal ulcer, and gender, residence, as well as number of patients hospitalized in the Almaty hospital №1 from 2009-2012, in order to learn about trends in the incidence of these diseases in Kazakhstan.

\section{MATERIAL AND METHODS}

In this work, patients $(\mathrm{n}=950)$ hospitalized with stomach and duodenal ulcers during the last three months of each year (October, November and December) in the four following years (2009-2012) were questioned. A questionnaire was handed out consisting of questions regarding the patient's residence (urban or rural), gender (female or male) and stomach or duodenal ulcer problems. In the analysis, a descriptive statistics method was used, and all derived data was made presentable as tables or figures. The study was conducted in the hospital located in the capital city.

\section{RESULTS AND DISCUSSION}

In Kazakhstan, slightly more people live in urban $(53.0 \%$ in 2008 and $54.7 \%$ in 2011) than in rural areas $(47.0 \%$ in 2008 and $45.3 \%$ in 2011) [9]. In our study, the patients hospitalized for suffering from stomach or duodenal ulcers were mostly from urban areas (68.33\%), only $31.67 \%$ were from rural areas (Fig. 1). A possible reason for this situation is that the larger numbers of urban patients may have better access to medical care. Indeed, very similar results were observed in Vietnam, where $59 \%$ of those infected with $H$. pylori lived in urban areas and only $31 \%$ were from rural areas [7]. In addition, it was noticed that more women (60.84\%) suffered from stomach or duodenal ulcers then men (39.16\%) (Fig. 2). This confirmed the hypothesis that more women suffer from ulcers [16]. Similar studies also showed that more females $(53 \%)$ are infected by H. pylori in Alaska [3] and Vietnam (H. pylori: $42.5 \%$ males and $57.5 \%$ females) [7]. One the other hand, a study performed by Schubert et al. [15] showed that more male suffered from duodenal ulcers.

Beyond the aforementioned, our work showed that considerably more patients suffered from stomach $(69.79 \%)$ than duodenal (30.21\%) ulcers (Fig. 3). The data in Figure 4 indicate that the number of patients suffering from duodenal ulcer is almost constant (about 20 per month) over the duration of the study. However, regarding stomach ulcer, the least number of patients suffering from this disease was noticed in December 2009 (31 patients), while the largest was seen in October and November, 2011 (68 and 67 patients, respectively). After a large decrease in the number of cases in November 2011, in the subsequent year of the study, in October, November and December 2012, the number of cases returned to a relatively high level (54-56 patients each month).

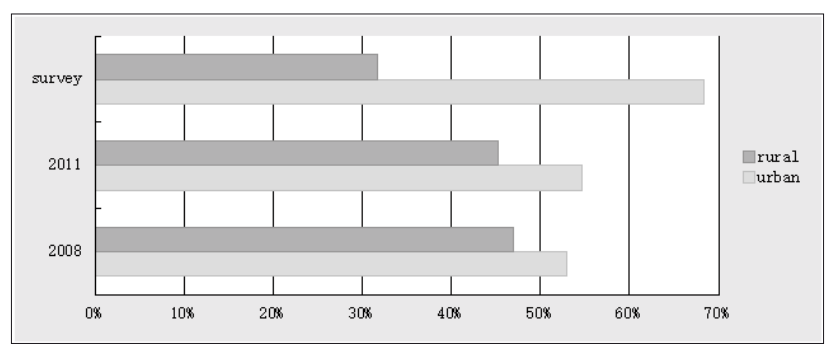

Figure 1. Kazakhstan demography divided into urban and rural areas

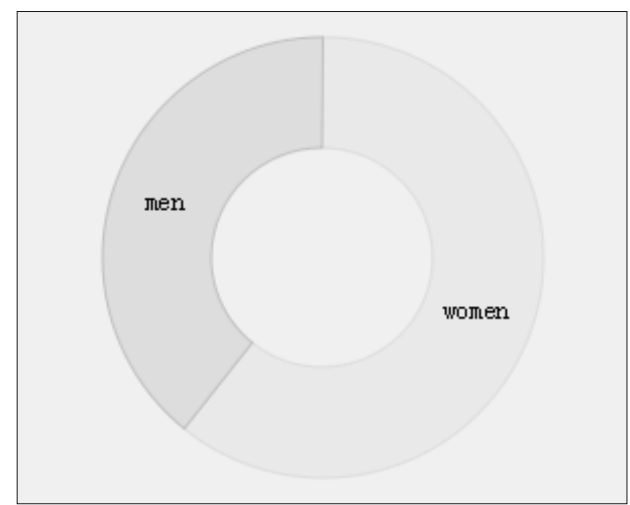

Figure 2. Patients with gastric or duodenal ulcers - based on gender

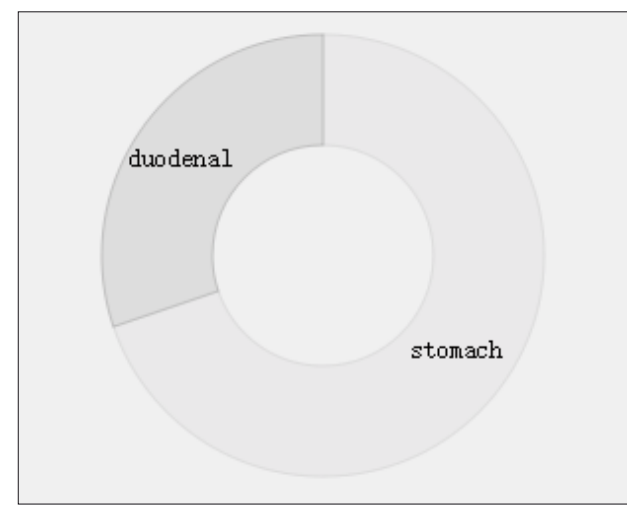

Figure 3. The incidence of gastric and duodenal ulcers

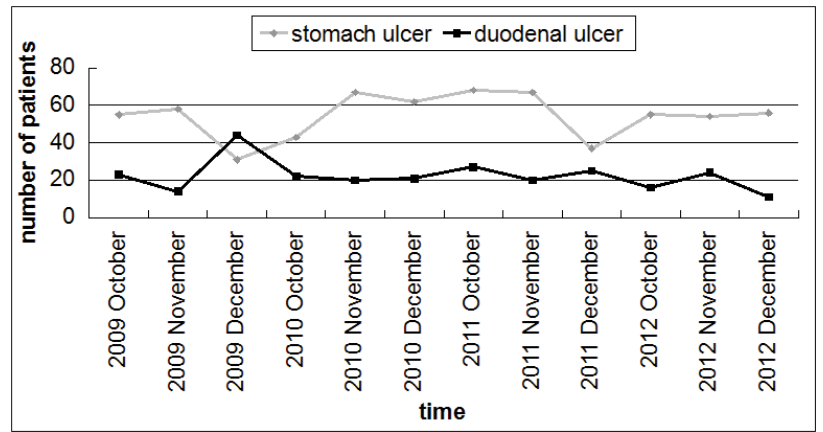

Figure 4. Number of patients admitted to hospital with gastric or duodenal ulcers - in the given months

The data gathered during the 4 years of the existence of the study, showed that the constant, relatively high amount of patients with ulcers is a serious problem in Kazakhstan. 
Yet, there is no appropriate procedure to reduce the number of patients suffering from ulcers. In most cases, such ulcers are connected with the presence of $H$. pylori. However, its diagnosis and total eradication from the human organism is difficult and expensive. A similar high number of patients infected with $H$. pylori and suffering from ulcers are seen in other Asian countries - such as Thailand [1], India [14], Japan and Korea [17]. In contrast, like studies performed in the United Kingdom between the years 1997-2005 have revealed that the care of peptic ulcers in Western Europe has improved. Within these years, the incidence of stomach ulcer has dropped from 1.1/1000/year in 1997, to $0.52 / 1000 /$ year in 2005 . This situation is most likely connected with a greater eradication of $H$. pylori, the frequent use of proton pump inhibitors and the use of less harmful non-steroidal anti-inflammatory drugs [4].

\section{REFERENCES}

1. Bodhidatta, L., et al.: Diagnosis of Helicobacter pylori infection in a developing country: comparison of two ELISA and a seroprevalence study. J. Infect. Dis., 168, 1551, 1993.

2. Brown L. M.: Helicobacter pylori: epidemiology and routes of transmission. Epidemiol. Rev., 2, 290, 2000.

3. Bruce M. G., et al.: Alaska sentinel surveillance for antimicrobial resistance in Helicobacter pylori isolates from Alaska native persons, 1999-2003. Helicobacter, 11, 583, 2006.

4. Cai S., et al: Uncomplicated peptic ulcer in the UK: trends from 1997 to 2005. Aliment. Pharm. Therap., 30, 1041, 2009.

5. Cotticelli L., et al.: Central serous chorioretinopathy and Helicobacter pylori. Eur. J. Ophthalmol., 16, 276, 2006.

6. Giusti C.: Association of Helicobacter pylori with central serous chorioretinopathy: Hypotheses regarding pathogenesis. Med. Hypotheses, 63, 525, 2004.
7. Hoang T. T. H., et al.: Sero-prevalance of Helicobacter pylori infection in urban and rural Vietnam. Clin. Diagn. Lab. Immun., $12,83,2005$

8. Hunt R. H., et al.: Helicobacter pylori in developing countries. http://www.worldgastroenterology.org/assets/downloads/en/pdf/ guidelines/11_helicobacter_pylori_developing_countries_en.pdf (cited: 13.03.2015 11:19)

9. Kazachstan in figures 2011, Astana $2012 \mathrm{http}: / / \mathrm{www} \cdot \mathrm{ecosn}$. org/Portals/0/Publications/Kazakhstan/Kazakhstan\%20in\%20 figures\%202011.pdf (cited: 24.03.2015 13:27).

10. Lau J. Y., et al.: Systematic review of the epidemiology of complicated peptic ulcer disease: incidence, recurrence, risk factors and mortality. Digestion, 84:105, 2011.

11. Marshall B.J. and Warren J. R.: The bacterium Helicobacter pylori and its role in gastritis and peptic ulcer disease. http://www. nobelprize.org/nobel_prizes/medicine/laureates/2005/press.html (cited: 13.03.2015 10:23)

12. MedlinePlus http://www.nlm.nih.gov/medlineplus/ency/ article/000206.htm (cited: 27.03.2015)

13. Prabhu V. and Shivani A: An overview of history, pathogenesis and treatment of perforated peptic ulcer disease with evaluation of prognostic scoring in adults. Ann. Med. Health Sci. Res., 4, 25, 2014.

14. Romero-Gallo J., et al.: Responses of endoscopy patient in Ladakh, India, to Helicobacter pylori whole-cell and CagA antigens. Clin. Diagn. Lab. Immunol., 9, 1315, 2002.

15. Schubert T. T, et al.: Ulcer risk factors: Interactions between Helicobacter pylori infection, nonsteroidal use, and age. Am. J. Med., 94, 416, 1993.

16. Thorsen K., et al.: Epidemiology of perforated peptic ulcer: Ageand gender-adjusted analysis of incidence and mortality. World J. Gastroenterol., 19, 349, 2013.

17. Youn H. S., et al.: Comparison of Helicobacter pylori infection between Fukuoka, Japan and Chinju, Korea. Helicobacter, 3, 11, 1998.

18. Zelickson M. S., et al.: Helicobacter pylori is not the predominant etiology for peptic ulcers requiring operation. Am. Surg., 77, 1059, 2011 